„In press“ version of: Steinhauer, K., Drury, J.E., Royle, P. \& Fromont, L.A. (2017). The priming of priming: Evidence that the N400 reflects context-dependent post-retrieval word integration in working memory. Neuroscience Letters, 651, 192-197. DOI: https://doi.org/10.1016/j.neulet.2017.05.007

\title{
The priming of priming: Evidence that the N400 reflects context- dependent post-retrieval word integration in working memory
}

\author{
Karsten Steinhauer ${ }^{1,2,}{ }^{*}$, Phaedra Royle ${ }^{2,3}$, John E. Drury ${ }^{4}$ and Lauren A. Fromont ${ }^{2,3}$ \\ ${ }^{1}$ School of Communication Sciences and Disorders, McGill University, Montreal, Canada \\ ${ }^{2}$ Centre for Research on Brain, Language and Music, Montreal, Canada \\ ${ }^{3}$ École d'orthophonie et d'audiologie Université de Montréal, Montréal, Canada \\ ${ }^{4}$ Department of Linguistics, Stony Brook University, Stony Brook, NY, USA \\ * Corresponding author: \\ Karsten Steinhauer, Ph.D. \\ Professor \\ McGill University \\ School of Communication Sciences \& Disorders \\ 2001 McGill College Ave. Unit 800 \\ Montreal, Quebec H3A-1G1; Canada. \\ Phone: 514-398-2413 \\ Fax: 514-398-8123 \\ Email: karsten.steinhauer@mcgill.ca
}

Keywords: N400, Semantic priming, Context effects, List effects, Relational priming, Analogical reasoning 


\begin{abstract}
Which cognitive processes are reflected by the N400 in ERPs is still controversial. Various recent articles (Lau et al., 2008; Brouwer et al., 2012) have revived the idea that only processes during word-retrieval (such as automatic spreading activation, ASA) are strongly supported, while post-lexical integrative processes are not. The present ERP study replicates a behavioral study by McKoon and Ratcliff (1995) who demonstrated that a prime-target pair such as finger - hand shows stronger priming when a majority of other pairs in the list share the analogous semantic relationship (here: part-whole), even at short stimulus onset asynchronies (250 ms). We created lists with four different types of semantic relationship (synonyms, part-whole, category-member, opposites) and compared priming for pairs in a consistent list with those in an inconsistent list as well as unrelated items. Highly significant N400 reductions were found for both relatedness priming (unrelated vs. inconsistent) and relational priming (inconsistent vs. consistent). These data are taken as strong evidence that N400 priming effects are not exclusively carried by ASA-

like mechanisms during lexical retrieval but also include post-lexical integration in working memory. The present findings will be linked to a neurocomputational model for relational reasoning (Knowlton et al., 2012) and to recent discussions of context-dependent conceptual activations (Yee \& Thompson-Schill, 2016).
\end{abstract}

\title{
1 Introduction
}

The N400 component is a negative-going brain wave in event-related potentials (ERP) peaking at approximately $400 \mathrm{~ms}$ after stimulus presentation and reflects the cost of lexicalsemantic processing [1]. Its amplitude seems to decrease with increasing predictability of the target word (cloze probability) and, possibly, ease of integration into the context. This pattern holds both for sentence contexts and for priming studies in which a semantically related prime word facilitates the recognition and processing of a given target word (e.g., doctor - nurse) compared to an unrelated prime (e.g., apple - nurse). From a psycholinguistic perspective, semantic priming constitutes an important experimental paradigm, because it advances our understanding of word recognition and of the lexicalsemantic network associated with the mental lexicon [2], but also because many recent approaches to sentence processing emphasize the role of expectancy based parsing [3, 4]. From a neurolinguistic perspective, ERP priming studies can help clarify the specific neurocognitive processes underlying the N400 component, one of the most important physiological correlates of real-time language processing.

\subsection{Priming mechanisms}

In the behavioural priming literature, three different priming mechanisms have been proposed [2, 5]: (1) Automatic spreading of activation (ASA) was the first such proposal [6] and assumes that semantically related 'word nodes' in long-term memory (LTM) are linked, such that activation of one node (e.g., doctor) spreads to related nodes (e.g., nurse) and pre-activates them, thereby facilitating subsequent lexical access. Given that ASA is viewed as fast, automatic and passive, semantic priming due to ASA is taken as mandatory 
at least at short stimulus onset asynchronies (SOAs) of less than $300 \mathrm{~ms}$ between prime and target, but it also seems to play a role at longer SOAs. (2) In contrast to ASA, the second priming mechanism is based on expectancy (prediction), is more controlled (i.e., subject to strategies), and usually gains influence at longer SOAs while it tends to be absent at short ones. Predicted target words are assumed to be active in working memory (WM) [7], such that this mechanism does not primarily operate within the semantic network in LTM, although the corresponding word nodes in the network may receive an additional (secondary) activation boost beyond ASA. (3) The third, and perhaps least understood, mechanism is semantic matching or semantic integration, which also takes place in WM, but only after both prime and target words have already been accessed (hence the term 'post-lexical integration'). For example, it explains the backward-priming phenomenon, i.e., faster response times for word pairs in which the second stimulus primes the first one, but not vice versa (e.g., box - lunch). Compared to the other two mechanisms, semantic matching is most likely to inherently provide some information about the type of semantic relationship between prime and target (e.g., synonyms vs. part-whole relationships).

\subsection{Priming effects on the N400}

To what extent the three proposed priming mechanisms contribute to N400 amplitude reduction has been controversial for more than twenty years. Automatic priming via ASA had been generally assumed, until Brown and Hagoort [8] reported that masked priming at short SOAs resulted in behavioral priming effects, but not in a reduction of the N400 amplitude. Similarly, Chwilla and colleagues [9] found that performing physical letter case discrimination during reading completely eliminated N400 priming effects even between strongly related prime-target pairs. The authors interpreted this finding as evidence that post-lexical semantic integration (and not ASA) underlies N400 priming effects, and were successfully prevented by their task. Alternatively, the nature of their task may not have promoted semantic network activation, thereby preventing ASA from taking place. Indeed, other researchers maintain that N400 priming effects are primarily (or even exclusively) driven by ASA. For example, Deacon and colleagues [10] and Kiefer [11] observed N400 priming effects with very short SOAs ( $<70 \mathrm{~ms})$ in a masked priming paradigm, suggesting that the underlying mechanism must have been ASA. Similarly, Brouwer and colleagues [12] suggest that the N400 is exclusively linked to lexical retrieval, while (post-lexical) semantic integration is supposed to be reflected by the P600 component. Moreover, as neuroimaging studies found consistent priming effects for the brain structure assumed to house the lexical semantic network (e.g., the posterior part of the middle temporal gyrus, pMTG), the influential review paper by Lau and colleagues [13] argued that retrievalrelated processes including ASA are likely to always contribute to N400 priming effects, irrespective of SOA duration. In contrast, they emphasize that the data reviewed "do not provide any conclusive evidence to support the integration account of the N400 effect" [p. 928], although they acknowledge this possibility cannot be ruled out by the data. Turning to future research, they suggest that "paradigms that selectively manipulate integrative processes at different levels of representation [...] will be needed” [p. 929].

A more recent ERP article by Lau et al. [7] extended the 2008 article by clarifying that activation-based N400 effects during initial word retrieval in LTM may also include 
prediction-based priming. The authors manipulated the proportion of semantically related prime-target pairs (10\% versus 50\%) in two experimental blocks, where the higher proportion should encourage participants to use strategies to predict the target word. Their $600 \mathrm{~ms}$ SOA was long enough for participants to profit from strategies. Indeed, while the low-proportion condition only showed the classical (presumably ASA-driven) N400 reduction between 300 and $500 \mathrm{~ms}$, in the high-proportion condition an additional - and earlier - ERP priming effect was found between 200 and $450 \mathrm{~ms}$. The latter also reduced the N400 amplitude and was attributed to higher target predictability. The authors argue that while WM-mediated contextual prediction mechanisms go beyond passive spreading activation (ASA), ultimately both types of priming "facilitate lexical access in exactly the same way and have the same neural effects in temporal cortex" (Ellen Lau, personal communication, February 2017), thereby reducing the N400. Thus, even though the observed prediction effects are said to involve WM, they do not seem to shed any light on the "integration account of N400 effects".

\subsection{Present study}

In order to fill this gap, our current ERP study aims at investigating the specific contribution of post-lexical semantic matching to N400 effects. The design was inspired by McKoon and Ratcliff's behavioral study [14], which tested various semantically related prime-target pair lists. Each list was dominated by a specific kind of semantic relationship (e.g., one list with synonyms such as far - distant or room - space, another list with partwhole relationships such as finger - hand or blade - knife, and yet other lists with opposites, category-member relationships, etc.). Importantly, they moved some of these highly related prime-target pairs from their original list into another list, e.g., a few synonyms into the part-whole list (and vice versa), and found that priming effects on these items were dramatically reduced or even absent. In other words, far seems to prime its synonym distant more strongly if a majority of other prime-target pairs in the list are also synonyms. Such a contextual effect is very difficult to explain in terms of ASA, for two reasons: First, ASA priming relies on node (word or concept) pre-activation, not on the links connecting them. Secondly, neither the activation levels of a prime target pair in LTM nor the semantic links between them should change just because other words being processed happen to have similar links between them. With long SOAs, McKoon and Ratcliff's contextual list effect could possibly be accounted for by prediction mechanisms (in line with [7]), but their SOA was $250 \mathrm{~ms}$ and thus arguably too short for expectancybased priming. In other words, their findings are likely to reflect priming due to postlexical integration. We predicted that a replication of their study with ERPs would reveal whether the N400 component does in fact reflect semantic integration (in addition to the established ASA and prediction-based priming effects). The present study was conducted in French. 


\section{Materials and Methods}

\subsection{Participants}

18 young francophone adults between 18 and 35 years of age, all native speakers of Quebec French with no history of neurological or language disorders, participated in the experiment. All participants were right-handed according to the Edinburgh handedness test [15]. Two were rejected from the analyses due to movement artefacts or excessive eyeblinks. The study was conducted in accordance with the Helsinki Declaration, and all participants read and signed an informed consent form. The protocol was approved by the McGill Faculty of Medicine Internal Review Board.

\subsection{Materials}

Four lists of 72 semantically related prime-target pairs were developed, one for the following 4 semantic relationships: 1 . Part-whole (metonyms): doigt - main 'finger hand'; 2. Member-category (hyponyms): marteau - outil 'hammer - tool'; 3. Synonyms: loin - distant 'far - distant'; and 4. Antonyms: rapide - lent 'fast - slow'. The different lists were then manipulated in order for each one to promote both a majority (consistent) and a minority (inconsistent) type of semantic relationship. To create inconsistent trials, nine of the 72 items in each list were swapped with items from the three other lists (three per list). In addition, nine unrelated prime-target pairs were added to each list, resulting in a total of 81 word pairs per list: 63 (78\%) consistent, 9 (11\%) inconsistent, and 9 (11\%) unrelated. Swapped items were rotated across subjects in order to avoid item effects. That is, we created eight different versions of the experiment (each version presented to 2 participants), such that all participants saw all 324 word pairs exactly once, but differed as to whether a given related pair was presented in its consistent list (a synonym in the list of synonyms) or in an inconsistent list (the same synonym in one of the other 3 lists). This ensured that all related word pairs contributed equally to both the consistent and the inconsistent condition. The trial order in each list (and all versions) was pseudorandomized to avoid that 2 inconsistent or 2 unrelated word pairs were presented in direct succession. List presentation order was counter-balanced across subjects (using a Latin Square design), each participant seeing all four consistent conditions in a blocked design, with a short break between two lists.

\subsection{Procedure}

Participants were seated in a sound-attenuating shielded booth and were presented with the four blocks of written word pairs. Each trial started with a centered fixation cross (600 ms), followed by a blank screen (200 ms), the prime word (200 ms), a blank screen (50 ms), the target word (200 ms), a blank screen (1000 ms), and a response prompt upon which the subject was instructed to judge if the two words were related (using mouse keys which were counter-balanced across subjects). Subsequently, subjects had 2,000 ms time to blink their eyes, after which a fixation cross started the next trial. Both the high proportion of semantically related word pairs (89\%) and the specific task were expected to encourage the use of strategies, including taking advantage of analogical semantic relationships among 
consistent list items. However, the short 250 ms prime-target SOA was too short to allow for expectancy-based priming.

\subsection{EEG recording}

EEG was continuously recorded (500 Hz sampling rate; Neuroscan Synamps2 amplifier) from 19 cap-mounted Ag/AgCl electrodes (Electro-Cap International) referenced to the right mastoid and placed according to the 10-20 System (impedance $<5 \mathrm{k} \Omega$ ). EOG was recorded from bipolar electrode arrays.

\subsection{Data analysis}

Acceptability judgment data were subjected to repeated-measures ANOVAs with factor CONDITION (3 levels: consistent, inconsistent, unrelated). EEG data were analyzed using EEProbe (ANT, The Netherlands). After data filtering (0.3-30 Hz bandpass) and artifact rejection, single subject averages were computed separately for target words for $1050 \mathrm{~ms}$ epochs beginning $300 \mathrm{~ms}$ prior to target word onset (-300 to $0 \mathrm{~ms}$ baseline). ERPs were quantified by amplitude average means in four consecutive $100 \mathrm{~ms}$ windows between 300 and 700 ms. To analyze ERP effects, repeated-measures ANOVAs with factor Condition (3 levels) were run separately for midline (with factor ELECTRODE: Fz/Cz/Pz/Oz) and 12 lateral electrodes (F3/4/7/8, C3/4, T3/4/5/6, P3/4) with topographical factors HEMISPHERE (left vs. right), LATERALITY (lateral vs. medial), and ANTPOST (3 levels: Anterior/Central/Posterior). Greenhouse-Geisser corrections were employed where applicable.

\section{Results}

\subsection{Behavioral Data}

The global ANOVA for correct responses revealed a significant main effect of CONDITION $(F(2,45)=5.33, p=0.008)$. Post-hoc tests involving Tukey multiple comparisons revealed that the scores in the UN(related) condition $(M=71, S D=45.41)$ were significantly lower than the CON(sistent) condition $(M=85.91, S D=34.79, p<0.01)$, and the IN(consistent) condition $(M=82.48, S D=38.04 p=0.05)$, whereas no difference was observed between the latter two $(p=0.77)$. The global ANOVA for RTs revealed only a non-significant trend for CONDITION $(F(2,45)=2.49, p=0.09)$.

\subsection{ERP results}

Figure 1 shows the ERPs in all three conditions time-locked to the onset of the target word (at $0 \mathrm{~ms}$ ). Whereas the UN condition elicited a large negativity that is most prominent between 300 and $500 \mathrm{~ms}$ (N400), targets preceded by a semantically related prime demonstrate a clear priming effect (reduced negativity) between 300 and $700 \mathrm{~ms}$ at all electrodes. Most importantly, this effect seems even larger in the CON than the IN condition, although this difference is slightly delayed (it starts around $400 \mathrm{~ms}$ ). The global ANOVAs including all three conditions revealed a significant CONDITION main effect 
between 300 and $400 \mathrm{~ms}$ at both midline $(F(1,15)=5.25, p<.05)$ and lateral electrodes $(F(1,15)=7.61, p<.01)$. In each of the three subsequent 100 -ms time windows between 400 and $700 \mathrm{~ms}$, the CONDITION main effect was even more significant, again both at midline electrodes (all F-values > 15.0, $p$ 's <.001) and at lateral electrodes (all F-values > $10.9, p$ 's $<.001)$. The only additional effect was a CONDITION*ANTPOST interaction at lateral electrodes for the two time windows $400-500 \mathrm{~ms}(F(2,30)=7.22, p<0.01)$ and 500 $600 \mathrm{~ms}(F(2,30)=5.60, p<.01)$. In order to distinguish between effects of relatedness (UN minus IN) and list consistency (IN minus CON), we conducted follow-up contrasts for the relevant conditions in each time window. These are summarized in Table $\mathbf{1}$ and in the next two sections. Difference waves that illustrate the relatedness and the consistency effect can be found in Figure 2, along with corresponding voltage maps.
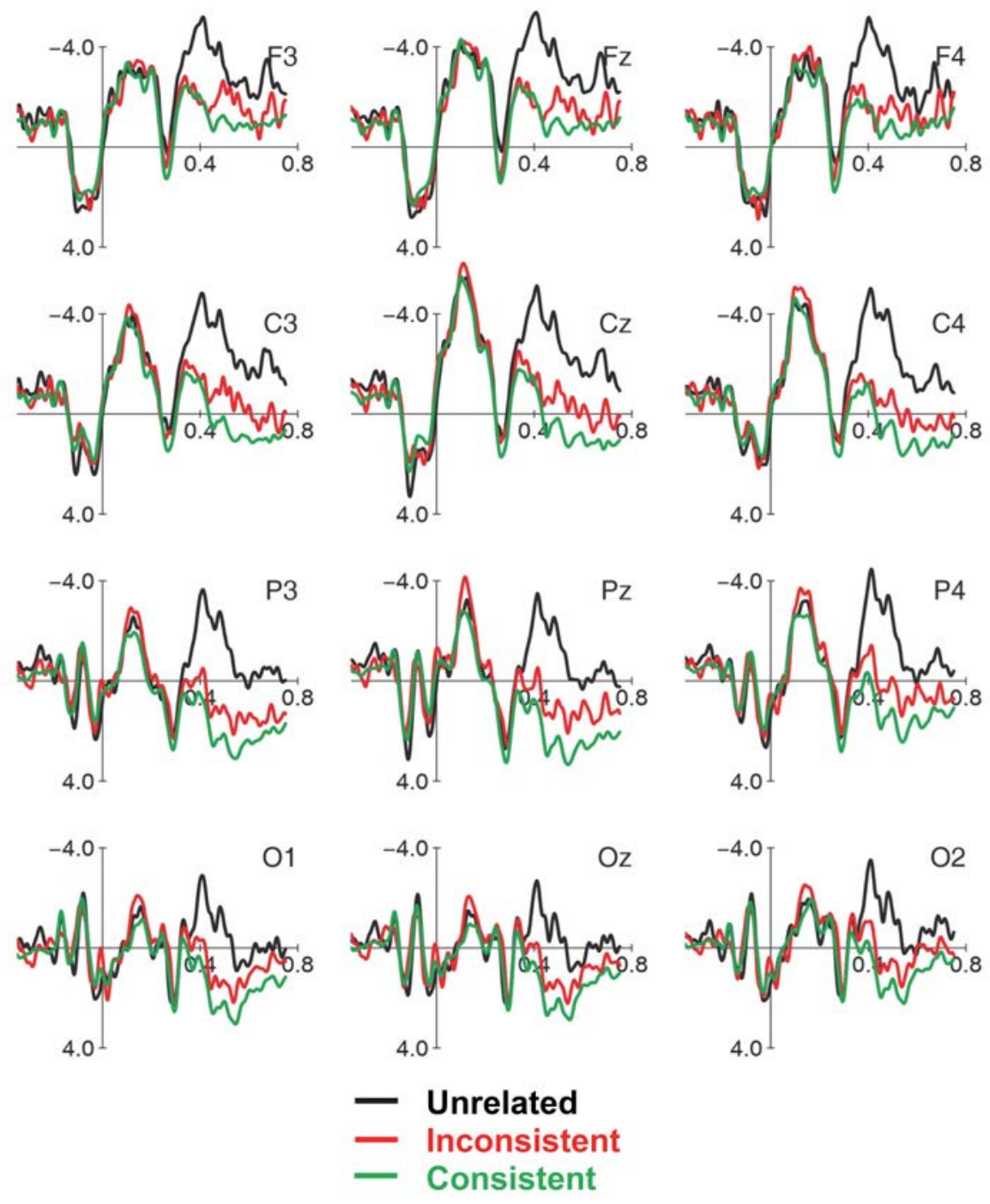

Fig 1: ERPs in all three conditions at 12 electrodes, time-locked to the target word (vertical line). While the inconsistent condition displays a typical N400 priming effect starting at $300 \mathrm{~ms}$, the consistent items show an additional N400 reduction starting around 400 ms. 


\section{Table 1}

Repeated measures ANOVA results for 'relatedness' and 'consistency' effects at midline and lateral electrodes, in $100 \mathrm{~ms}$ time-windows from 300 to $700 \mathrm{~ms}$.

\begin{tabular}{|c|c|c|c|c|c|}
\hline & $\mathrm{df}$ & $300-400$ & $400-500$ & $500-600$ & $600-700$ \\
\hline \multicolumn{6}{|c|}{ UNRELATED - INCONSISTENT (relatedness effect) } \\
\hline \multicolumn{6}{|c|}{ Midline } \\
\hline Condition & 1,15 & $5.69 *$ & $25.68 * * *$ & $14.52 * *$ & $8.57 *$ \\
\hline \multicolumn{6}{|l|}{ Lateral } \\
\hline Condition & 1,15 & $9.09 * *$ & $33.66^{* * *}$ & $7.80 *$ & $5.70 *$ \\
\hline Cond.*Lat. & 1,15 & $5.27 *$ & $16.35 * *$ & $22.65 * * *$ & $21.09 * * *$ \\
\hline Cond.*Ant. & 2,30 & & $6.39 *$ & $5.02 *$ & \\
\hline \multicolumn{6}{|c|}{ INCONSISTENT - CONSISTENT (consistency effect) } \\
\hline \multicolumn{6}{|l|}{ Midline } \\
\hline Condition & 1,15 & & $8.22 *$ & $9.29 * *$ & $5.16^{*}$ \\
\hline \multicolumn{6}{|l|}{ Lateral } \\
\hline Condition & 1,15 & & $8.23 * *$ & $9.17 * *$ & $4.00 !$ \\
\hline Cond.*Lat.*Ant. & 2,30 & & & & $3.73 *$ \\
\hline
\end{tabular}

\subsubsection{Relatedness effects: contrasting unrelated and inconsistent word pairs}

As shown in Table 1, the IN condition elicited a significantly smaller negativity than the UN condition in all 4 time windows, both at midline and lateral electrodes. Additional CONDITION*LATERALITY interactions (also in all 4 time windows) reflect the fact that the priming effects are more prominent at medial electrodes. Finally, a significant CONDITION*ANTPOST interaction in the two middle time windows (400-600 ms) point to a centro-parietal focus for the relatedness effect (see voltage maps in Figure 2). Overall, the relatedness effect was largest and most significant between 400 and 500 ms. 


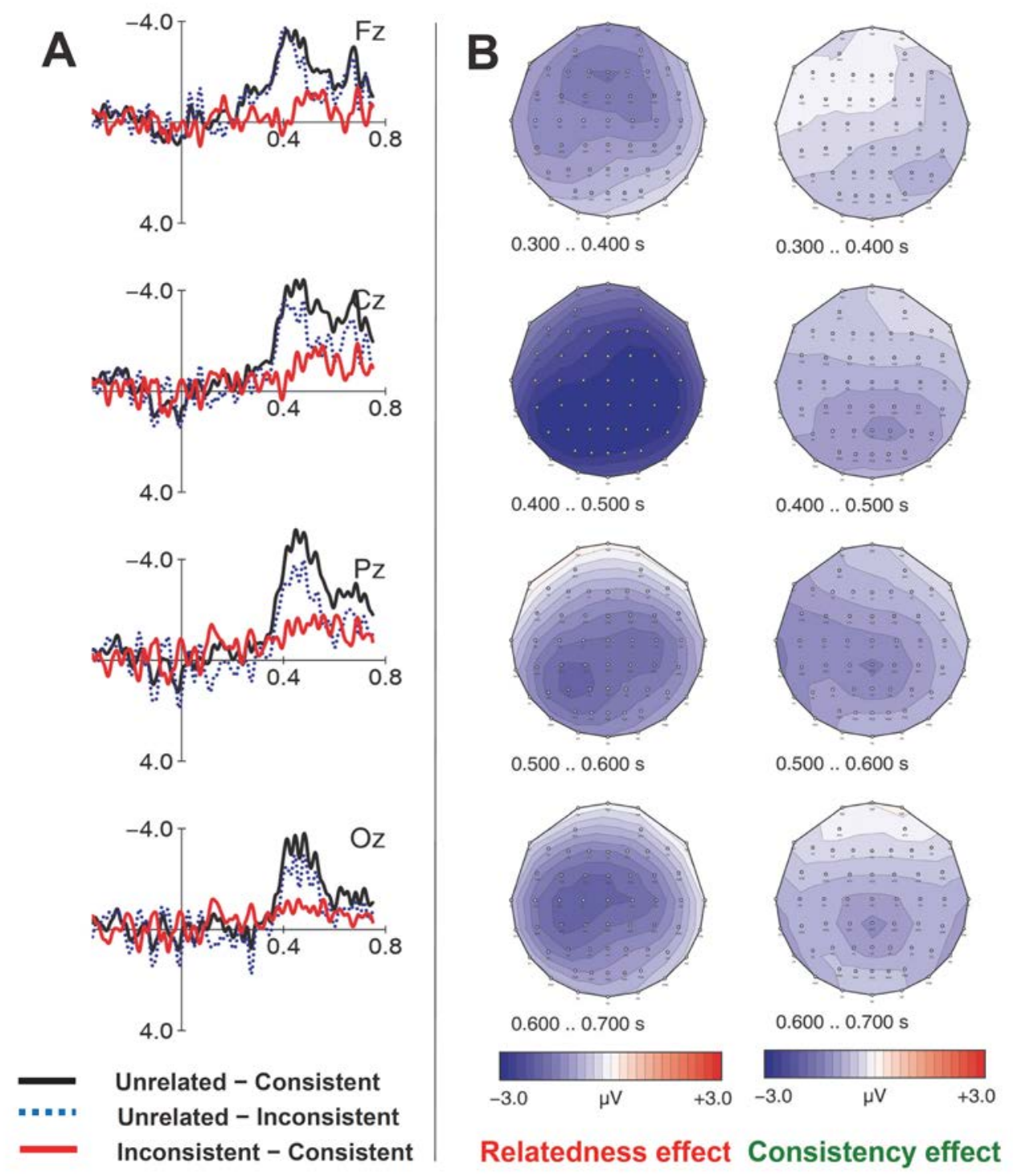

Fig 2: (A) Difference waves for the two priming effects at midline electrodes (for more electrodes see Supplementary Material). The black line represents the sum of the relatedness effect (blue) and the consistency effect (red). (B) Voltage maps illustrate the scalp distribution of the relatedness and the consistency effect per time window.

\subsubsection{Consistency effects: contrasting inconsistent and consistent word pairs}

Unlike the relatedness effect, the consistency effect (contrasting IN and CON conditions) reached significance only after $400 \mathrm{~ms}$ (see Table 1). At midline electrodes it remained significant until $700 \mathrm{~ms}$, whereas the ANOVA at lateral electrodes revealed significant CONDITION main effects only until $600 \mathrm{~ms}$, as well as a marginal effect between 600 and 700 ms. In this latter time window, a CONDITION*LATERALITY*ANTPOST interaction reflected the finding that the late portion of the priming effect was restricted to parietal electrodes near the midline. 


\section{Discussion}

The present ERP data replicate and extend previous behavioural findings by McKoon and Ratcliff [14] and demonstrate that the N400 amplitude for semantically related primetarget pairs (e.g., finger-hand) is substantially attenuated when most other word pairs in the list have the same type of semantic relationship (e.g., part-whole). In other words, for listconsistent items the priming effect itself seems to be enhanced (or 'primed'). If N400 priming effects relied exclusively on ASA along the hard-wired connections between lexical or conceptual nodes in LTM, our finding could only be explained if we assume that specific semantic relationship types themselves are represented by a corresponding node (which would imply a different architecture from traditional semantic network models [6]). But even then, the $100 \mathrm{~ms}$ delay between ASA-based semantic priming (affecting the N400 no later than $300 \mathrm{~ms}$ ) and relational (or analogical) priming (after $400 \mathrm{~ms}$ ) would remain mysterious. One could also argue that our effects are due to prediction mechanisms. However, we believe that our 250 ms SOA is too short to support this account. To test its assumptions, it should be possible to manipulate the SOA such that either only the N400 or both the N250 and the N400 would be affected. For example, results from [7] (including N250 reductions around $200 \mathrm{~ms}$ ) should be fully replicable with SOAs of $450 \mathrm{~ms}$ (see Supplementary Materials for details on this argument).

Our alternative interpretation of a post-lexical (post-retrieval) relational priming mechanism in working memory (rather than LTM) avoids these problems and is, importantly, strongly supported by the literature. First, most review papers on the neural N400 generators point to the prefrontal cortex (PFC) and inferior frontal cortex (IFC), in addition to the temporal lobe [16-18]. Second, there is an increasing literature emphasizing the critical role of context for conceptual processing and priming (including task effects), perhaps most prominently summarized by Yee and Thompson-Schill (2016) [19]. Third, relatively recent research on 'relational priming' and ‘analogical reasoning' has reported behavioral facilitation effects (glass - eye $\rightarrow$ copper - horse) [20, 21], linked analogical reasoning to both working memory and PFC structures using event-related fMRI [22, 23], and found preliminary evidence for $\mathrm{N} 400$ attenuations for analogical reasoning applied to two word pairs (e.g., open/close - push/pull) as well [24].

Arguably the most compelling neuro-computational model for analogical priming and reasoning to date was proposed by Knowlton and colleagues in 2012 [23], based on the "learning and interference in schemas and analogies" framework (LISA) by Hummel and Holyoak (1997) [25]. The authors suggest that a 'transient representation of a structure unit' that captures the core role relationship between elements in focus (such as part-whole) is 'formed in prefrontal cortex' (i.e., in working memory) and can be used to identify the systematic correspondence between the elements of two analogs that are active in working memory (analogical mapping). Importantly, neurons in the PFC (but not in the inferiotemporal cortex) were indeed found to specifically respond to the type of relationship (not the stimuli) and to be extremely flexible: A neuron's response to a given stimulus can change based on the relevant relationship even from trial to trial. In other words, the extended LISA framework provides a sophisticated account for our data and strongly suggest a post-lexical checking process (analogical mapping) in working memory. 
We propose that all related word pairs in our study resulted in classical priming in LTM (mediated by ASA and reducing the N400 amplitude around $300 \mathrm{~ms}$ ). However, in addition, our participants also generated temporary structural units in working memory (referred to as 'proxy unit' in LISA $[23,25]$ ) that represented the role relations specific for a given list (e.g., part-whole, category-member, etc.). These units in WM allowed them to semantically integrate list-consistent items much faster than semantically related word pairs whose relationship did not match the 'proxy unit' specifications. As these processes were post-lexical (and took place in WM), the onset of this priming effect was delayed compared to ASA and reduced the N400 only after 400 ms. This might suggest that early N400 priming effects may be generally due to lexical pre-activation mechanisms (such as ASA and prediction) whereas later N400 effects after $400 \mathrm{~ms}$ are at least partly influenced by post-lexical integration (see [26] for a related notion of staged N400 effects). The observation that both N400 priming effects (in LTM and WM) lasted beyond $500 \mathrm{~ms}$ in our study (and many other N400 studies, e.g., [7, 27]) is likely related to the specific task. While our study only provides one example of post-lexical integration processes resulting in N400 effects, we believe this is a very common phenomenon that plays a crucial role in everyday sentence processing.

Our results, along with those of previous studies, also allow us to draw a relatively precise picture of whether and when different priming mechanisms affect the N400 component.

- Prediction-based priming: $200-450 \mathrm{~ms}$. The earliest priming effect after target word onset affects both the more frontal N250 (formal phonological or orthographic priming; [7, 26, 27]) and the centro-parietal N400. Arguably, prediction-based priming requires a sufficiently long SOA between prime and target (600 ms in Lau et al. [7]; but see Supplementary Materials).

- ASA-driven priming / word retrieval : 300-500 ms. Similar to most ERP studies on lexical-semantic processing, our relatedness effect (UN - CON) started around $300 \mathrm{~ms}$. As this effect often ends around $500 \mathrm{~ms}$. We believe that the longer duration in our study (until $700 \mathrm{~ms}$ ) is task related.

- Post-lexical integration / post-retrieval mapping: after 400 ms. Our present data show that context-driven post-lexical integration can take place no later than 400 ms after target word onset and modulates the N400 within its classical time window (300-500 ms). This is problematic for models that postulate the N400 reflects only processes during word retrieval.

Acknowledgments: We thank Ellen Lau for very constructive comments on the first version of our manuscript. This work was supported by grants awarded to the first author by the Canada Research Chair program (Project \# 950-209843), the Canada Foundation for Innovation (Project \# 201876), and the Social Sciences and Humanities Research Council (410-2007-1501 and 435-2013-2052). 


\section{References}

[1] M. Kutas, K.D. Federmeier, Thirty Years and Counting: Finding Meaning in the N400 Component of the Event-Related Brain Potential (ERP), Annual Review of Psychology 62 (2011) 621-647.

[2] J.H. Neely, Semantic priming effects in visual word recognition: A selective review of current findings and theories. In: D. Besner, G. Humphreys (Eds.), The Psychology of Learning and Motivation: Advances in research and theory, Vol. 24, Academic Press, San Diego, CA, 1991, pp. 207-247.

[3] R. Levy, Expectation-based syntactic comprehension, Cognition 106 (2008) 11261177.

[4] E. Lau, C. Stroud, S. Plesch, C. Phillips, The Role of Structural Prediction in Rapid Syntactic Analysis, Brain and Language 98 (2006) 74-88.

[5] M.S. Franklin, J. Dien, J.H. Neely, E. Huber, L.D. Waterson, Semantic priming modulates the N400, N300, and N400RP, Clinical Neurophysiology 118 (2007) 1053-1068.

[6] A.M. Collins, E.F. Loftus, A spreading-activation theory of semantic processing, Psychological Review 82 (1975) 407-428.

[7] E.F. Lau, P.J. Holcomb, G.R. Kuperberg, Dissociating N400 effects of prediction from association in single word contexts, Journal of Cognitive Neuroscience 25 (2013) 484-502.

[8] C. Brown, P. Hagoort, The Processing Nature of the N400: Evidence from Masked Priming, Journal of Cognitive Neuroscience 5 (1993) 34-33.

[9] D.J. Chwilla, C.M. Brown, P. Hagoort, The N400 as a function of the level of processing, Psychophysiology 32 (1995) 274-285.

[10] D. Deacon, S. Hewitt, C.-M. Yang, M. Nagata, Event-related potential indices of semantic priming using masked and unmasked words: evidence that the N400 does not reflect a post-lexical process, Cognitive Brain Research 9 (2000) 137-146.

[11] M. Kiefer, The N400 is modulated by unconsciously perceived masked words: Further evidence for an automatic spreading activation account of N400 priming effects, Cognitive Brain Research 13 (2002) 27-39.

[12] H. Brouwer, H. Fitz, J. Hoeks, Getting real about Semantic Illusions: Rethinking the functional role of the P600 in language comprehension, Brain Research 1446 (2012) 127-143.

[13] E.F. Lau, C. Phillips, D. Poeppel, A cortical network for semantics: (de)constructing the N400, Nature Reviews Neuroscience 9 (2008) 920-933.

[14] G. McKoon, R. Ratcliff, Conceptual combinations and relational contexts in free association and in priming lexical decision and naming, Psychonomic Bulletin and Review 2 (1995) 527-533.

[15] R.C. Oldfield, The Assessment and Analysis of handedness: The Edinburgh Inventory, Neuropsychologia 9 (1971) 97-115.

[16] C. Van Petten, B.J. Luka, Neural localization of semantic context effects in electromagnetic and hemodynamic studies, Brain and Language 97 (2006) 279_ 293.

[17] B. Maess, C.S. Herrmann, A. Hahne, A. Nakamura, A.D. Friederici, Localizing the distributed language network responsible for the N400 measured by MEG during 
auditory sentence processing, Brain Research 1096 (2006) 163-172.

[18] E. Halgren, R.P. Dhond, N. Christensen, C. Van Petten, K. Marinkovic, J.D.

Lewine, A.M. Dale, N400-like Magnetoencephalography Responses Modulated by Semantic Context, Word Frequency, and Lexical Class in Sentences, NeuroImage 17 (2002) 1101-1116.

[19] E. Yee, S.L. Thompson-Schill, Putting concepts into context, Psychonomic Bulletin \& Review 23 (2016) 1015-1027.

[20] Z. Estes, L.L. Jones, Priming via relational similarity: A COPPER HORSE is faster when seen through a GLASS EYE, Journal of Memory and Language 55 (2006) 89-101.

[21] B.A. Spellman, K.J. Holyoak, R.G. Morrison, Analogical priming via semantic relations, Memory \& Cognition 29 (2001) 383-393.

[22] S.A. Bunge, C. Wendelken, D. Badre, A.D. Wagner, Analogical Reasoning and Prefrontal Cortex: Evidence for Separable Retrieval and Integration Mechanisms, Cerebral Cortex 15 (2005) 239-249.

[23] B.J. Knowlton, R.G. Morrison, J.E. Hummel, K.J. Holyoak, A neurocomputational system for relational reasoning, Trends in Cognitive Sciences 16 (2012) 373-381.

[24] R.G. Morrison, M.J. Kmiecik, K.L. Bharani, When Analogy is Like Priming: The N400 in Verbal Analogical Reasoning. Cognitive Neuroscience Society Annual Meeting, Chicago, IL, 2012.

[25] J.E. Hummel, K.J. Holyoak, Distributed representations of structure: A theory of analogical access and mapping, Psychological Review 10 (1997) 427-466.

[26] T. Brothers, T.Y. Swaab, M.J. Traxler, Effects of prediction and contextual support on lexical processing: Prediction takes precedence, Cognition 136 (2015) 135-149.

[27] P. Royle, J.E. Drury, N. Bourguignon, K. Steinhauer, The temporal dynamics of inflected word recognition: A masked ERP priming study of French verbs, Neuropsychologia 50 (2012) 3542-3553. 